\title{
The Extended Fourier Transform for 2D Spectral Estimation
}

\author{
Geoffrey S. Armstrong and Vladimir A. Mandelshtam \\ Chemistry Department, University of California_Irvine, Irvine, California 92697-2025 \\ E-mail: garmstro@uci.edu; mandelsh@uci.edu
}

Received February 28, 2001; revised August 10, 2001

We present a linear algebraic method, named the eXtended $F$ ourier Transform (XFT), for spectral estimation from truncated time signals. The method is a hybrid of the $d$ iscrete Fourier transform (DFT) and the regularized resolvent $t$ ransform (RRT) (J. Chen et al., J. Magn. Reson. 147, 129-137 (2000)). Namely, it estimates the remainder of a finite DFT by RRT. The RRT estimation corresponds to solution of an ill-conditioned problem, which requires regularization. The regularization depends on a parameter, $q$, that essentially controls the resolution. By varying $q$ from 0 to $\infty$ one can "tune" the spectrum between a high-resolution spectral estimate and the finite DFT. The optimal value of $q$ is chosen according to how well the data fits the form of a sum of complex sinusoids and, in particular, the signal-to-noise ratio. B oth 1D and 2D XFT are presented with applications to experimental N MR signals. (c) 2001 A cademic Press

Key Words: extended Fourier transform; spectral estimation; resolution enhancement; regularized resolvent transform; filter diagonalization method.

\section{INTRODUCTION}

In this paper we are concerned with the problem of spectral estimation from truncated 1D and 2D time signals, which is the central problem of data processing in a number of experiments based on Fourier transform (FT) spectroscopy and, in particular, NMR spectroscopy.

Consider a 1D time signal $c(n):=c(n \tau)(n=0, \ldots, N-1)$ that has been sampled discretely on an equidistant set of time points. The infinite time discrete Fourier transformation $\left(\mathrm{DFT}_{\infty}\right)$ of $c(n)$ is then defined as

$$
I(s)=\sum_{n=0^{+}}^{\infty} c(n) z^{-n}:=\sum_{n=0}^{\infty}\left(1-\frac{\delta_{n 0}}{2}\right) c(n) z^{-n},
$$

with $z=e^{-i \tau s}$. (The "shortcut" $\sum_{n=0^{+}}$, meaning that the first term in the sum is multiplied by $\frac{1}{2}$, will be used throughout the paper. This multiplication corrects the error due to the discretization of the half-line Fourier integral.) A finite DFT can be used to estimate $I(s)$ :

$$
I(s) \approx \operatorname{DFT}_{N}(s)=\sum_{n=0^{+}}^{N-1} c(n) z^{-n}
$$

$\mathrm{DFT}_{N}$ gives spectral resolution $\delta s \sim 2 \pi / N \tau$. This slow convergence behavior is known as the FT uncertainty principle. For example, peaks that are separated by less than $2 \pi / N \tau$ cannot be discerned. The DFT resolution can also be affected by an artifact known as Gibbs oscillations. This arises from an abrupt truncation of the signal, giving small wiggles in the baseline of the spectrum, affecting the resolution by obscuring weak signals. Gibbs oscillations are normally suppressed by weighting the time signal with an appropriate apodization function, allowing the signal to smoothly decay to 0 within the sampling interval. However, this also results in an additional, slight broadening of the lines, further reducing the resolution.

A number of methods that try to overcome the DFT limitations have been suggested in the past for high-resolution spectral estimation from truncated data sets. Typically, the higher resolution is obtained by incorporating some additional information about the signal that is ignored in the DFT processing. Among such methods, the most relevant to the present framework are the autoregression (AR) techniques (see, e.g., Ref. (1) and references therein), i.e., based on the assumption that there exists a number $K$, so that the data satisfies the AR form

$$
c(n)=\sum_{p=1}^{K} a_{p} c(n-p) .
$$

If $N \geq 2 K$, one can now solve Eq. [3] for the prediction coefficients $a_{p}$ and then use them directly (see, e.g., $(2,3)$ ) to extend the available time domain data to times longer than the sampling interval. The extended signal is then transformed with a DFT. Note that Eq. [3] is also commonly referred to as the linear prediction (LP) assumption. It can be shown that for a general case it is equivalent to assuming that the signal is composed of $K$ complex sinusoids with complex frequencies $\omega_{k}$ and amplitudes $d_{k}$,

$$
c(n)=\sum_{k=1}^{K} d_{k} u_{k}^{n}
$$

with $u_{k}=e^{-i \tau \omega_{k}}$. A number of parameter estimation methods have been developed to solve the harmonic inversion problem (HIP) for the unknowns $u_{k}$ and $d_{k}$ as defined by Eq. [4] (see, e.g., 
Refs. $(1,4-8)$ and references therein). The computed spectral parameters $u_{k}$ and $d_{k}$ can be used to construct the spectrum $I(s)$. The important common aspect of most such methods is that they may be formulated purely within the linear algebraic framework, making them computationally very efficient compared to, e.g., a general nonlinear parametric fit problem. However, a common difficulty, encountered in the techniques for solving either the spectral or parameter estimation problem, is their notorious ill-defined nature and various instabilities, especially in cases when the data do not fit the AR assumption [3]. Moreover, the difficulties increase for large and/or multidimensional data sets.

The filter diagonalization method (FDM), introduced recently (8-15) belongs to the class of AR techniques, although it is derived using a different assumption about the data, formulated in terms of the quantum time autocorrelation function with an effective (complex symmetric) evolution operator $\hat{U}$ and initial state $\Phi(9)$ :

$$
c(n)=\left(\Phi\left|\hat{U}^{n}\right| \Phi\right) .
$$

Here $(a \mid b)=(b \mid a)$ denotes the complex symmetric inner product. Equation [5] turns out to be equivalent to both Eqs. [3] and [4] if we assume $K$ to be the rank of $\hat{U}$ with eigenvalues $u_{k}$. If we now define the corresponding eigenfunctions as $\Upsilon_{k}$, the amplitudes become $d_{k}=\left(\Phi \mid \Upsilon_{k}\right)^{2}$. Thus, once a matrix representation of $\hat{U}$ is obtained in terms of the available data points $c(n)$, the spectral parameters $u_{k}$ and $d_{k}$ are estimated from the corresponding eigenvalue problem. The key difference between FDM and most other AR algorithms is the use of a Fourier subspace (9) that reduces a typically large linear algebraic problem (defined by the data size) to a small one. As such the actual power of FDM reveals when the data sets are large and in the cases of multidimensional spectral analysis, where the quantum mechanical framework becomes very convenient to devise efficient multidimensional linear algebraic algorithms. Note though that other Fourier subspace methods exist for 1D spectral analysis such as LP-ZOOM (5) or beamspacing (see Ref. (16) and references therein), although they are not commonly used for the NMR data processing. (For a comparison of LP-ZOOM with 1D FDM see Ref. (11).)

In another linear algebraic spectral estimation technique, arising from FDM and called regularized resolvent transform (RRT) (17), the solution of either the AR Eq. [3] or the HIP [4] is avoided. The spectrum is estimated directly using the resolvent formula derived by substituting the assumption [5] into Eq. [1]:

$$
\begin{aligned}
I(s)=\sum_{n=0^{+}}^{\infty}\left(\Phi\left|\hat{U}^{n}\right| \Phi\right) z^{-n} & =\left(\Phi\left|\left\{\sum_{n=0}^{\infty}(\hat{U} / z)^{n}-\frac{1}{2}\right\}\right| \Phi\right) \\
& =\left(\Phi\left|\left\{\frac{1}{1-\hat{U} / z}-\frac{1}{2}\right\}\right| \Phi\right) .
\end{aligned}
$$

When the data fit the Lorentzian assumption (i.e., one of the equations [3], [4], or [5], which are all equivalent) well, both FDM and RRT give essentially identical and excellent results.
However, in the case when this assumption is not satisfied, such as for very short data sets and/or noisy signals, they may become unstable or difficult to use. A common source of difficulty is the need to fiddle with the parameters of the method to achieve an acceptable compromise between high spectral resolution (possibly with spurious or poorly converged peaks) and low resolution (with all artifacts suppressed). Figure 1 illustrates the problems associated with spectral estimation. The 2D RRT spectral estimate of a sufficiently large data set $\left(N_{1} \times N_{2}=16 \times 300\right)$ is very well converged and gives a superior resolution to the finite $2 \mathrm{D}$ DFT spectral estimate. Quite unfortunately, 2D RRT has sudden convergence. That is, when the data is truncated below some critical size (e.g., $N_{1} \times N_{2}=8 \times 32$ ), it fails catastrophically, producing unacceptable lineshapes and some missing peaks. The DFT of the same truncated data gives a uniform low-resolution spectral estimate, although producing strong Gibbs oscillations. Because the finite DFT spectral estimate is not governed by any assumptions, it is straightforward to use, is well understood, and, as such, is most commonly used. It is often desirable to have a controllable procedure that corrects the DFT by, e.g., suppressing the Gibbs oscillations and improving the resolution, at least for the peaks with Lorentzian lineshapes. This strategy is, in principle, well known and can be implemented in the framework of most nonlinear spectral or parameter estimators. For example, LP is most commonly used to extend the data to longer times followed by DFT processing. However, because of various difficulties associated with appearance in the line list of complex poles, $u_{k}$, outside the unit circle, $\left|u_{k}\right|>1$, the extrapolation may be difficult to implement because of the exponential instabilities. Several prescriptions for combining DFT with FDM have been suggested previously $(9,14)$. However, those appeared to be difficult to extend to a $2 \mathrm{D}$ case, in particular, because the spectral parameters in 2D are much harder to obtain, and, thus, it is hard to reconstruct a $2 \mathrm{D}$ signal. In this paper we propose a procedure, named the e $X$ tended $F$ ourier $T$ ransform (XFT), that can be used to correct the 1D and 2D DFT spectral estimates in a controllable fashion by adding a, generally, small correction term computed by RRT. That is, an XFT spectral estimate may be written in the form

$$
I(s) \approx \mathrm{XFT}_{N}=\mathrm{DFT}_{N}+\Delta_{N},
$$

where the second term is an RRT-based estimate of the $N$ th DFT remainder,

$$
\Delta_{N}=\sum_{n=N}^{\infty} c(n) z^{-n} .
$$

The XFT, therefore, extracts spectral features lost by limitations of the finite DFT. The RRT requires regularization, depending on a parameter, $q$, that controls the artifact suppression and level of resolution. When $q=\infty$ the correction, $\Delta_{N}$, vanishes (low resolution); finite values of $q$ give nonzero correction (high resolution), provided the data have not decayed to zero during the acquisition period. Using exact arithmetic for data sets 

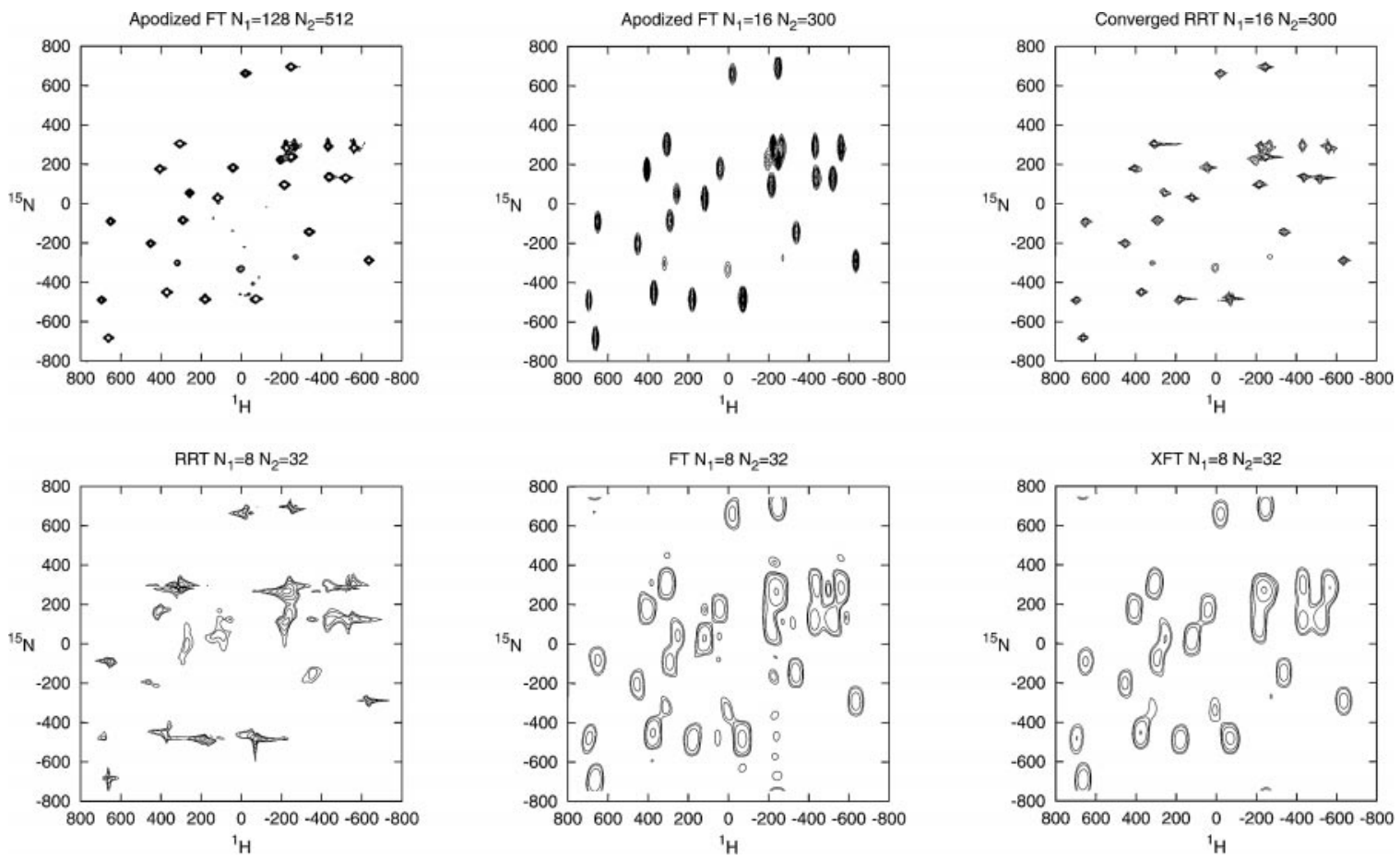

FIG. 1. Chemical shift correlation spectra of the metalloprotein rubredoxin. The RRT for the severely truncated data gives nonuniform lineshapes and omits some of the features. The DFT for the severely truncated data has correct lineshapes and features, but has artifacts due to the truncation. The XFT is able to yield the DFT spectrum without artifacts even when the high-resolution estimation fails. In this example, the spectra have been calculated with spectral widths of $1500 \times 1600 \mathrm{~Hz}$, with 200 spectral points in each dimension. The RRT and XFT spectra for the truncated data were both obtained using $K_{\text {win }}=32$. The RRT of the longer signal had $K_{\text {win }}=80$.

satisfying, e.g., the form [4] with $N \geq 2 K$ and infinitesimally small values of $q$, the XFT estimate gives the exact result. It should be emphasized that $\Delta_{N}$ is not computed by processing any kind of residual signal or by extrapolating the data. Because extrapolation is avoided, the common exponential instability does not occur in XFT. An example of 2D XFT in action is shown in Fig. 1. It is clear that, although the resolution enhancement of the spectrum, compared to DFT, is not enormous, in this case of severely truncated data, XFT does suppress the DFT artifacts and reveals some small spectral features that are missing in the DFT spectrum. At the same time, the XFT lineshapes remain well behaved. In the next sections we derive the 1D and 2D XFT and examine their behavior using both 1D and 2D NMR data.

\section{1D XFT}

The correction term [8] is estimated by analogy with Eq. [6] using Eq. [5],

$$
\begin{aligned}
\Delta_{N}=\sum_{n=N}^{\infty}\left(\Phi\left|(\hat{U} / z)^{n}\right| \Phi\right) & =z^{-N}\left(\Phi_{M}\left|\sum_{n=0}^{\infty}(\hat{U} / z)^{n}\right| \Phi_{N-M}\right) \\
& =\left(\Phi_{M}\left|\frac{z^{-N}}{1-\hat{U} / z}\right| \Phi_{N-M}\right),
\end{aligned}
$$

for any $M \leq N$, where we have defined the state vectors $\Phi_{n}:=$ $\hat{U}^{n} \Phi$. It is convenient to choose $N=2 M$. We can now evaluate the corresponding matrix element of the resolvent in Eq. [9] using the basis $\left\{\Phi_{n}\right\}(n=0, \ldots, M-1)$. (A more numerically efficient basis is a Fourier-type basis, but this will be discussed later.) Given the assumption [5], the elements of the square $M \times$ $M$ overlap matrix $\left[\mathrm{U}_{0}\right]_{n m}:=\left(\Phi_{n} \mid \Phi_{m}\right)$, the evolution operator matrix $\left[\mathrm{U}_{1}\right]_{n m}:=\left(\Phi_{n}|\hat{U}| \Phi_{m}\right)$ and the column $M \times 1$ vector $\left[\mathrm{C}_{m}\right]_{n}:=z^{-m}\left(\Phi_{n} \mid \Phi_{m}\right)$ are given in terms of the available signal points $c(n)$ :

$$
\begin{aligned}
{\left[\mathbf{U}_{1}\right]_{n m} } & =c(n+m+1), \\
{\left[\mathbf{U}_{0}\right]_{n m} } & =c(n+m), \\
{\left[\mathbf{C}_{m}\right]_{n} } & =z^{-m} c(n+m) .
\end{aligned}
$$

Now by replacing $\Phi_{M}$ and $(1-\hat{U} / z)$ in Eq. [9] by their matrix representations [10], the correction term becomes

$$
\Delta_{N} \approx \mathbf{C}_{M}^{\mathrm{T}} \mathbf{R}^{-1} \mathbf{C}_{M}
$$

with the $M \times M$ matrix-pencil $\mathbf{R}=\mathbf{U}_{0}-\mathbf{U}_{1} / z$ and $N=2 M$.

Numerical solution of Eq. [11] may scale as $\sim N^{3}$ if one does not take advantage of the special Hankel structure of $\mathbf{R}$. A Fourier basis has proven to be very efficient for such problems $(9,10)$. 
A small Fourier subspace $\left\{\tilde{\Phi}_{j}\right\}$ of size $K_{\text {win }} \ll M$ is constructed by choosing $K_{\text {win }}$ complex numbers on the unit circle $y_{j}=$ $e^{-i \tau \varphi_{j}}\left(j=1, \ldots, K_{\mathrm{win}}\right)$ and using

$$
\tilde{\Phi}_{j}=\sum_{n=0}^{M-1} y_{j}^{-n} \Phi_{n}
$$

(In this paper the tilde over vectors or matricies will symbolize the expression in the Fourier basis.) The efficiency of the Fourier basis depends on how the points $\varphi_{j}$ are chosen. In most cases an equidistant grid is effective, with spacing

$$
\Delta \varphi=\frac{2 \pi}{\aleph M \tau}
$$

and adjusting parameter $\aleph \geq 1$ (e.g., one can use $\aleph=1.2$ ). Since the basis is localized in the frequency domain, the diagonal elements of the matricies will dominate, and the off-diagonal elements will drop off as a sinc function. This allows an accurate estimate of the inverse $\mathbf{R}^{-1}$ in a small subspace, leading to numerically cheap matrix inversion. A further gain may be achieved by using a multiscale Fourier basis (14) with a nonuniform set of $\varphi_{j}$, containing information about the entire spectrum rather than just the spectral window. The multiscale basis is not used here for simplicity.

The matrix elements of the $K_{\text {win }} \times K_{\text {win }}$ square matrices $\tilde{\mathbf{U}}_{1}$ and $\tilde{\mathbf{U}}_{0}$ can be computed using (10)

$$
\left[\tilde{\mathbf{U}}_{\boldsymbol{p}}\right]_{j j^{\prime}}=\hat{S} \sum_{\sigma=0,1} \frac{(-1)^{\sigma}\left(y_{j} / y_{j^{\prime}}\right)^{\sigma M}}{1-y_{j} / y_{j^{\prime}}} \sum_{n=\sigma M}^{(\sigma+1)(M-1)} y_{j}^{-n} c(n+p),
$$

where $\hat{S}$ defines the symmetrization operator over the variables $y_{j}$ and $y_{j^{\prime}}$,

$$
\hat{S} g\left(y_{j}, y_{j^{\prime}}\right)=g\left(y_{j}, y_{j^{\prime}}\right)+g\left(y_{j^{\prime}}, y_{j}\right)
$$

For $j=j^{\prime}$ we have

$$
\left[\tilde{\mathbf{U}}_{p}\right]_{j j}=\sum_{n=0}^{2 M-2} y_{j}^{-n}(M-|M-n-1|) c(n+p) .
$$

The $m$ th state vector $\Phi_{m}$ in the Fourier basis is a $K_{\text {win }} \times 1$ column vector with elements

$$
\left[\tilde{\mathbf{C}}_{m}\right]_{j}=z^{-m} \sum_{n=0}^{M-1} y_{j}^{-n} c(n+m) .
$$

Now defining the $K_{\text {win }} \times K_{\text {win }}$ matrix-pencil $\tilde{\mathbf{R}}=\tilde{\mathbf{U}}_{0}-\tilde{\mathbf{U}}_{1} / z$ in the Fourier basis, the correction term becomes

$$
\Delta_{N} \approx \tilde{\mathbf{C}}_{M}^{\mathrm{T}} \tilde{\mathbf{R}}^{-1} \tilde{\mathbf{C}}_{M}
$$

Since a small subspace, rather than the complete basis $\left\{\Phi_{n}\right\}$, is used to evaluate the resolvent, the expression [18] must be viewed as an approximation to Eq.[11] with convergence parameter $K_{\text {win }}$. However, an acceptable convergence is usually achieved for sufficiently small sizes in the range $K_{\text {win }} \sim 10$ 100. Moreover, in the Fourier basis, the matrices are much less ill-conditioned, which makes them easier to handle numerically.

Just like in RRT (17), numerical evaluation of the resolvent in Eq. [18] requires regularization. For example, in the case when Eq. [5] is satisfied exactly (i.e., the Lorentzian assumption is exact) for the signal $c(n)$, the rank of $\mathbf{R}$ is $K$ if $M>K$, so the full $M \times M$ matrix $\mathbf{R}$ is singular and, therefore, $\mathbf{R}^{-\mathbf{1}}$ does not exist. In a Fourier basis representation with small $K_{\text {win }} \ll K$, the $K_{\text {win }} \times K_{\text {win }}$ matrix $\tilde{\mathbf{R}}$ will not be exactly singular. Moreover, in the presence of noise it would unlikely be singular for any $K_{\text {win }}$. However, it can still be ill-conditioned. There are several methods to deal with this situation (18), such as Tikhonov regularization $(17,19)$, but in this paper we consider the singularvalue decomposition (SVD). Although the latter is numerically more expensive than the former, it can still lead to a numerical saving when results at several regularization levels are needed. The SVD of the square matrix $\tilde{\mathbf{R}}$ is defined by

$$
\tilde{\mathbf{R}}=\mathbf{V}^{\dagger} \boldsymbol{\Sigma} \mathbf{W}
$$

with unitary matrices $\mathbf{V}$ and $\mathbf{W}$ and $\boldsymbol{\Sigma}=\operatorname{diag}\left(\sigma_{n}\right)$, real and diagonal; $\mathbf{V}^{\dagger}$ defines the Hermitian conjugate of $\mathbf{V}$. Since we can now write $\tilde{\mathbf{R}}^{-1}=\mathbf{W}^{\dagger} \boldsymbol{\Sigma}^{-1} \mathbf{V}$, the problem then becomes that of regularizing the diagonal matrix $\boldsymbol{\Sigma}^{-1}$. Here the truncated SVD (i.e., setting $\left[\Sigma_{q}^{-1}\right]_{n n}=0$ for very small singular values $\sigma_{n}$ below a certain threshold) is not recommended. Instead, a more uniform regularization may be employed using, for example,

$$
\left[\Sigma_{q}^{-1}\right]_{n n}= \begin{cases}\sigma_{n}^{-1}, & \text { if } \sigma_{n}>q \\ q^{-1}, & \text { otherwise }\end{cases}
$$

or

$$
\left[\Sigma_{q}^{-1}\right]_{n n}=\frac{\sigma_{n}}{\sigma_{n}^{2}+q}
$$

In this paper we employed Eq. [21], while we were informed recently that Eq. [20] could be more effective (20).

Regularization leads to the ultimate well-behaved expression to evaluate the correction in the 1D XFT,

$$
\Delta_{N} \approx \tilde{\mathbf{C}}_{M}^{\mathrm{T}} \mathbf{W}^{\dagger} \boldsymbol{\Sigma}_{q}^{-1} \mathbf{V} \tilde{\mathbf{C}}_{M}
$$

which is one of the main results of this paper.

The properties of the DFT term used in Eq. [7] may not be ideal: the DFT is calculated for a truncated data set and is not apodized. For this reason, the Gibbs oscillations in the spectrum are quite intense. These artifacts are compensated for by the correction in the XFT which, in turn, must be large and oscillatory. 
Although in our numerical tests this did not cause problems, potentially, it can be a source of instability. For this reason it may be beneficial to calculate a correction to the already apodized DFT spectrum, so that the large oscillations need not be compensated for. An example of such procedure is given in the Appendix.

\subsection{Numerical Example: $1 D X F T$}

The XFT of an experimental NMR data set can be examined to determine the role of each term. This is best accomplished in the $1 \mathrm{D}$ case as the correction to the baseline is evident. In Fig. 2 we show an interesting spectral region of $12 \mathrm{H}$-pyrido[1,2- $a: 3,4-$ $b$ ]diinole processed by DFT and XFT. The DFT was calculated using both $N=32000$ and $N=5000$ data points for comparison (upper traces). The lower three traces were computed using only 5000 of the available data points, which is short enough that the DFT spectrum results in some truncation artifacts, such as Gibbs oscillations, and insufficient resolution to resolve the doublets present in the DFT using 32,000 points. The correction (the bottom trace) has oscillations equal and opposite to the oscillations in the DFT. When added together these cancel, giving the correct baseline in the XFT spectrum. Furthermore, the correction also contains features that improve the resolution of the DFT, revealing some splittings that could not be obtained with

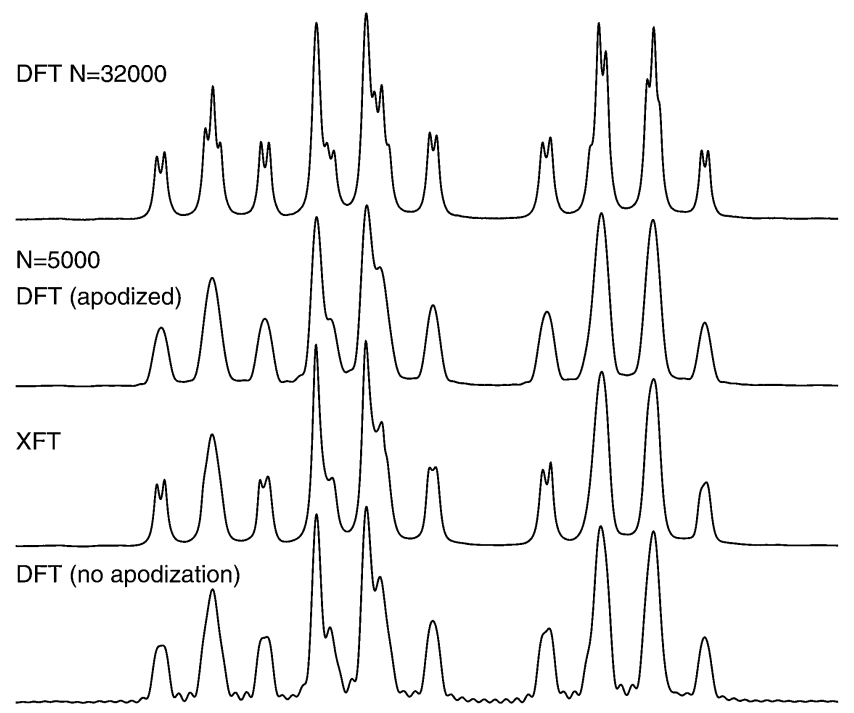

Correction

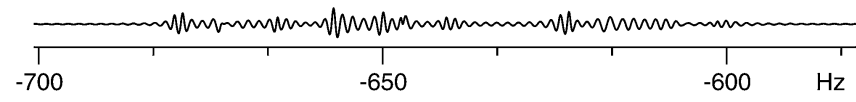

FIG. 2. An interesting region of the $1 \mathrm{D}$ NMR spectrum of $12 H$-pyrido[1,2$a: 3,4-b$ ]diinole, processed by the XFT using a truncated $(N=5000)$ data set. The figure illustrates the behavior of each term involved in the calculation of the XFT. The XFT is obtained by adding together the unapodized DFT term and the correction term calculated with a regularization parameter of $q$ that is optimized for this case. The correction term exactly cancels the Gibbs oscillations present in the nonapodized DFT. It also adds small terms to increase the resolution of some peaks, allowing some doublet structures to be identified. For comparison the apodized DFT spectra for $N=32000$ and $N=5000$ are presented.

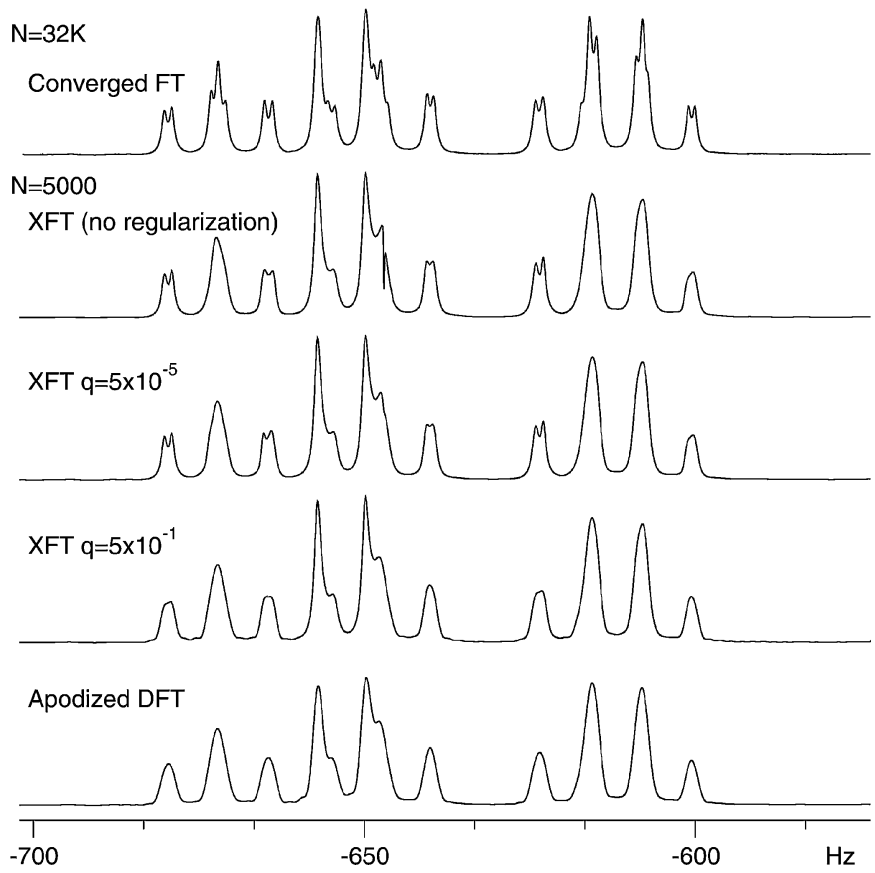

FIG . 3. The XFT spectra generated for a range of regularization parameter $q$ are compared to the DFT spectrum using the same number of data points $(N=5000)$. The latter was apodized with a cosine weighting function. The fully converged DFT spectrum using the entire data set is also shown. As $q$ increases, the XFT loses resolution, but still contains as much information as the DFT. In this case, the optimal value for $q$ is around $1 \times 10^{-5}$, where the small splittings can be resolved with artifacts of the nonlinear processing suppressed.

the truncated DFT alone. In this example the regularization parameter $q$ was optimized for maximum resolution enhancement. It should be noted that an optimal $q$ is roughly proportional to the factor $\mathcal{N}=N \sum_{n=0}^{N-1}|c(n)|$, so it is convenient to consider the scaled quantity $q \rightarrow q / \mathcal{N}$, which has a sensible order.

Now we examine the behavior of the XFT as a function of $q$. For very large $q$, the regularization parameter will dominate the resolvent and the correction will be zero. In this case, the spectrum will be identical to the DFT. When $q$ is very small, the correction term will be nonzero, yielding a high-resolution spectral estimate similar to the RRT (17) spectral estimate. For intermediate values of $q$, XFT will generally give different results from the RRT.

The effect of changing $q$ is demonstrated in Fig. 3. For no regularization $(q=0)$, an artifact is present at $-650 \mathrm{~Hz}$, which is removed by performing the calculation with $q=5 \times 10^{-5}$. When $q$ is set quite high $(q=0.5)$, the XFT becomes essentially equivalent to the apodized DFT. In this manner, even if the XFT cannot increase the resolution, it can still smooth the DFT, removing the Gibbs oscillations.

\section{2D XFT}

Although the 1D XFT is interesting for evaluating the successes and limitations of the method, it is the multidimensional 
generalization of XFT that is most useful. The simple reason is that there is usually no acquisition time limitation in 1D NMR experiments. In 2D, however, the difficulties associated with the data truncation are much more pronounced. As shown in Fig. 1 the high-resolution techniques, such as RRT, can be used quite successfully with signals severely truncated along the interferometric dimensions, provided the total size, $N_{1} \times N_{2}$, of the 2D data array is sufficiently large. When $N_{1} \times N_{2}$ is too small, RRT may fail catastrophically. XFT is manifestly a much less aggressive method as it tries to correct the DFT spectrum in a controllable fashion, sometimes giving only marginal improvement, but sometimes leading to a significant resolution enhancement.

We consider a 2D time signal on an evenly spaced 2D time grid $c\left(n_{1}, n_{2}\right):=c\left(n_{1} \tau_{1}, n_{2} \tau_{2}\right)$. The goal is then to estimate the infinite time 2D DFT spectrum using the scheme

$$
\begin{aligned}
I\left(s_{1}, s_{2}\right) & =\sum_{n_{1}=0^{+}}^{\infty} \sum_{n_{2}=0^{+}}^{\infty} z_{1}^{-n_{1}} z_{2}^{-n_{2}} c\left(n_{1}, n_{2}\right) \\
& =\operatorname{DFT}_{N_{1} N_{2}}+\Delta_{1}+\Delta_{2}+\Delta_{3}
\end{aligned}
$$

with $z_{1}=e^{-i \tau_{1} s_{1}}$ and $z_{2}=e^{-i \tau_{2} s_{2}}$. The finite 2D DFT and the three correction terms arise from breaking the infinite $2 \mathrm{D}$ summation domain into four rectangular regions:

$$
\begin{aligned}
\operatorname{DFT}_{N_{1} N_{2}} & =\sum_{n_{1}=0^{+}}^{N_{1}-1} \sum_{n_{2}=0^{+}}^{N_{2}-1} z_{1}^{-n_{1}} z_{2}^{-n_{2}} c\left(n_{1}, n_{2}\right) \\
\Delta_{1} & =\sum_{n_{1}=0^{+}}^{N_{1}-1} \sum_{n_{2}=N_{2}}^{\infty} z_{1}^{-n_{1}} z_{2}^{-n_{2}} c\left(n_{1}, n_{2}\right) \\
\Delta_{2} & =\sum_{n_{1}=N_{1}}^{\infty} \sum_{n_{2}=0^{+}}^{N_{2}-1} z_{1}^{-n_{1}} z_{2}^{-n_{2}} c\left(n_{1}, n_{2}\right) \\
\Delta_{3} & =\sum_{n_{1}=N_{1}}^{\infty} \sum_{n_{2}=N_{2}}^{\infty} z_{1}^{-n_{1}} z_{2}^{-n_{2}} c\left(n_{1}, n_{2}\right) .
\end{aligned}
$$

Generalizing the 1D quantum ansatz [5] the 2D signal is assumed to be generated by two commuting complex symmetric evolution operators $\hat{U}_{1}$ and $\hat{U}_{2}$ :

$$
c\left(n_{1}, n_{2}\right)=\left(\Phi\left|\hat{U}_{1}^{n_{1}} \hat{U}_{2}^{n_{2}}\right| \Phi\right) .
$$

Now using $2 M_{1}=N_{1}$ and $2 M_{2}=N_{2}$ and defining $\Phi_{n_{1}, n_{2}}:=$ $\hat{U}_{1}^{n_{1}} \hat{U}_{2}^{n_{2}} \Phi$, by analogy with Eq. [9], we can write

$$
\begin{aligned}
\Delta_{1}= & \sum_{n_{1}=0^{+}}^{M_{1}-1} z_{1}^{-n_{1}}\left(\Phi_{n_{1}, M_{2}}\left|\frac{z_{2}^{-2 M_{2}}}{1-\hat{U}_{2} / z_{2}}\right| \Phi_{0, M_{2}}\right) \\
& +\sum_{n_{1}=0}^{M_{1}-1} z_{1}^{-n_{1}}\left(\Phi_{n_{1}, M_{2}}\left|\frac{z_{1}^{-M_{1}} z_{2}^{-2 M_{2}}}{1-\hat{U}_{2} / z_{2}}\right| \Phi_{M_{1}, M_{2}}\right),
\end{aligned}
$$

$$
\begin{aligned}
\Delta_{2}= & \sum_{n_{2}=0^{+}}^{M_{2}-1} z_{2}^{-n_{2}}\left(\Phi_{M_{1}, n_{2}}\left|\frac{z_{1}^{-2 M_{1}}}{1-\hat{U}_{1} / z_{1}}\right| \Phi_{M_{1}, 0}\right) \\
& +\sum_{n_{2}=0}^{M_{2}-1} z_{2}^{-n_{2}}\left(\Phi_{M_{1}, n_{2}}\left|\frac{z_{2}^{-M_{2}} z_{1}^{-2 M_{1}}}{1-\hat{U}_{1} / z_{1}}\right| \Phi_{M_{1}, M_{2}}\right), \\
\Delta_{3}= & \left(\Phi_{M_{1}, M_{2}}\left|\frac{z_{1}^{-2 M_{1}}}{1-\hat{U}_{1} / z_{1}} \frac{z_{2}^{-2 M_{2}}}{1-\hat{U}_{2} / z_{2}}\right| \Phi_{M_{1}, M_{2}}\right) .
\end{aligned}
$$

The arising resolvent matrix elements can now be evaluated on the basis $\Phi_{n_{1}, n_{2}}$ of size $M_{1} \times M_{2}$. However, as in the 1D case, it is advantageous to implement a Fourier basis. The latter is introduced by selecting a $K_{1 \text { win }} \times K_{2 \text { win }}=K_{\text {win }}$ grid of points $\left(\varphi_{1 j}, \varphi_{2 j}\right)$ in a chosen $2 \mathrm{D}$ frequency window with spacings given by Eq. [13],

$$
\tilde{\Phi}_{j}=\sum_{n_{1}=0}^{M_{1}-1} \sum_{n_{2}=0}^{M_{2}-1} y_{1 j}^{-n_{1}} y_{2 j}^{-n_{2}} \Phi_{n_{1}, n_{2}},
$$

with $y_{1 j}=e^{-i n \tau_{1} \varphi_{1 j}}$ and $y_{2 j}=e^{-i n \tau_{2} \varphi_{2 j}}$. The square complex symmetric matrices of $\hat{U}_{1}, \hat{U}_{2}$ and the identity operator (the overlap matrix) in this basis are defined by $\tilde{\mathbf{U}}_{1}, \tilde{\mathbf{U}}_{2}$, and $\tilde{\mathbf{U}}_{0}$, accordingly. Their matrix elements are given here without derivation (see Ref. (11)). To consolidate the expressions, three new data sets are defined:

$$
\begin{aligned}
& c_{0}\left(n_{1}, n_{2}\right):=c\left(n_{1}, n_{2}\right), \\
& c_{1}\left(n_{1}, n_{2}\right):=c\left(n_{1}+1, n_{2}\right), \\
& c_{2}\left(n_{1}, n_{2}\right):=c\left(n_{1}, n_{2}+1\right) .
\end{aligned}
$$

The matrix elements of the $U$ matrices for both $y_{1 j} \neq y_{1 j^{\prime}}$ and $y_{2 j} \neq y_{2 j^{\prime}}$ can be computed by

$$
\begin{aligned}
{\left[\mathbf{U}_{l}\right]_{j j^{\prime}}=} & \hat{S}_{1} \sum_{\sigma_{1}=0,1} \frac{(-1)^{\sigma_{1}}\left(y_{1 j} / y_{1 j^{\prime}}\right)^{\sigma_{1} M_{1}}}{1-y_{1 j} / y_{1 j^{\prime}}} \\
& \times \hat{S}_{2} \sum_{\sigma_{2}=0,1} \frac{(-1)^{\sigma_{2}}\left(y_{2 j} / y_{2 j^{\prime}}\right)^{\sigma_{2} M_{2}}}{1-y_{2 j} / y_{2 j^{\prime}}} \\
& \times \sum_{n_{1}=\sigma_{1} M_{1}}^{\left.\sigma_{1}+1\right)\left(M_{1}-1\right)} \sum_{n_{2}=\sigma_{2} M_{2}}^{\left(\sigma_{2}+1\right)\left(M_{2}-1\right)} y_{1 j}^{-n_{1}} y_{2 j}^{-n_{2}} c_{l}\left(n_{1}, n_{2}\right),
\end{aligned}
$$

where $\hat{S}_{1}$ and $\hat{S}_{2}$ define the symmetrization operators over the corresponding pairs of variables as, e.g.,

$$
\hat{S}_{1} g\left(y_{1 j}, y_{1 j^{\prime}}\right)=g\left(y_{1 j}, y_{1 j^{\prime}}\right)+g\left(y_{1 j^{\prime}}, y_{1 j}\right) .
$$


For $y_{1 j}=y_{1 j^{\prime}}$ and $y_{2 j} \neq y_{2 j^{\prime}}$ we have

$$
\begin{aligned}
{\left[\mathbf{U}_{l}\right]_{j j^{\prime}}=} & \hat{S}_{2} \sum_{\sigma_{2}=0,1} \frac{(-1)^{\sigma_{2}}\left(y_{2 j} / y_{2 j^{\prime}}\right)^{\sigma_{2} M_{2}}}{1-y_{2 j} / y_{2 j^{\prime}}} \\
& \times \sum_{n_{1}=0}^{2 M_{1}-2} \sum_{n_{2}=\sigma_{2} M_{2}}^{\left(\sigma_{2}+1\right)\left(M_{2}-1\right)} y_{1 j}^{-n_{1}} y_{2 j}^{-n_{2}} c_{l}\left(n_{1}, n_{2}\right) \\
& \times\left(M_{1}-\left|M_{1}-n_{1}-1\right|\right),
\end{aligned}
$$

which can trivially be rewritten for the symmetric case of $y_{1 j} \neq$ $y_{1 j^{\prime}}$ and $y_{2 j}=y_{2 j^{\prime}}$. For the case of both $y_{1 j}=y_{1 j^{\prime}}$ and $y_{2 j}=$ $y_{2 j^{\prime}}$, i.e., the diagonal elements of the $U$ matrices, we have

$$
\begin{aligned}
{\left[\mathbf{U}_{l}\right]_{j j}=} & \sum_{n_{1}=0}^{2 M_{1}-2} \sum_{n_{2}=0}^{2 M_{2}-2} y_{1 j}^{-n_{1}} y_{2 j}^{-n_{2}} c_{l}\left(n_{1}, n_{2}\right) \\
& \times\left(M_{1}-\left|M_{1}-n_{1}-1\right|\right)\left(M_{2}-\left|M_{2}-n_{2}-1\right|\right) .
\end{aligned}
$$

The $U$ matrices are used to construct the matrix pencils

$$
\tilde{\mathbf{R}}_{l}=\tilde{\mathbf{U}}_{0}-\tilde{\mathbf{U}}_{l} / z_{l}, \quad(l=1,2) .
$$

Now define the $K_{\text {win }} \times 1$ column vectors $\tilde{\mathbf{C}}_{m_{1}, m_{2}}$ with elements $\left[\tilde{\mathbf{C}}_{m_{1}, m_{2}}\right]_{j}:=z_{1}^{-m_{1}} z_{2}^{-m_{2}}\left(\tilde{\Phi}_{j} \mid \Phi_{m_{1}, m_{2}}\right)$, which can be computed using

$$
\begin{aligned}
{\left[\tilde{\mathbf{C}}_{m_{1}, m_{2}}\right]_{j}=} & z_{1}^{-m_{1}} z_{2}^{-m_{2}} \\
& \times \sum_{n_{1}=0}^{M_{1}-1} \sum_{n_{2}=0}^{M_{2}-1} y_{1 j}^{-n_{1}} y_{2 j}^{-n_{2}} c\left(n_{1}+m_{1}, n_{2}+m_{2}\right) .
\end{aligned}
$$

In addition define the column $K_{\text {win }} \times 1$ vectors $\tilde{\mathbf{C}}_{\mathrm{FT}, m_{2}}$ and $\tilde{\mathbf{C}}_{m_{1}, \mathrm{FT}}$ obtained from $\tilde{\mathbf{C}}_{m_{1}, m_{2}}$ by summation along one of the dimensions,

$$
\begin{aligned}
\tilde{\mathbf{C}}_{\mathrm{FT}, m_{2}} & =\sum_{m_{1}=0}^{M_{1}-1} \tilde{\mathbf{C}}_{m_{1}, m_{2}}, \\
\tilde{\mathbf{C}}_{m_{1}, \mathrm{FT}} & =\sum_{m_{2}=0}^{M_{2}-1} \tilde{\mathbf{C}}_{m_{1}, m_{2}} .
\end{aligned}
$$

The numerical expressions for the correction terms in the Fourier basis can finally be written

$$
\begin{aligned}
\Delta_{1} & =\left(\tilde{\mathbf{C}}_{0, M_{2}}+\tilde{\mathbf{C}}_{M_{1}, M_{2}}\right)^{\mathrm{T}} \tilde{\mathbf{R}}_{2}^{-1} \tilde{\mathbf{C}}_{\mathrm{FT}, M_{2}}-\frac{1}{2} \tilde{\mathbf{C}}_{0, M_{2}}^{\mathrm{T}} \tilde{\mathbf{R}}_{2}^{-1} \tilde{\mathbf{C}}_{0, M_{2}}, \\
\Delta_{2} & =\left(\tilde{\mathbf{C}}_{M_{1}, 0}+\tilde{\mathbf{C}}_{M_{1}, M_{2}}\right)^{\mathrm{T}} \tilde{\mathbf{R}}_{1}^{-1} \tilde{\mathbf{C}}_{M_{1}, \mathrm{FT}}-\frac{1}{2} \tilde{\mathbf{C}}_{M_{1}, 0}^{\mathrm{T}} \tilde{\mathbf{R}}_{1}^{-1} \tilde{\mathbf{C}}_{M_{1}, 0}, \\
\Delta_{3} & =\tilde{\mathbf{C}}_{M_{1}, M_{2}}^{\mathrm{T}} \tilde{\mathbf{R}}_{1}^{-1} \tilde{\mathbf{U}}_{0} \tilde{\mathbf{R}}_{2}^{-1} \tilde{\mathbf{C}}_{M_{1}, M_{2}} .
\end{aligned}
$$

Equations [23]-[36] constitute the main result of this paper. Armed with these equations we can construct the 2D XFT.
Although the above derivation may appear quite tedious, the result itself is straightforward: the arrays entering Eq. [36] are all obtained from the data $c\left(n_{1}, n_{2}\right)$ by simple linear transformations involving DFT. Just as in the 1D case, numerical evaluation of the resolvents $\tilde{\mathbf{R}}_{1}^{-1}$ and $\tilde{\mathbf{R}}_{2}^{-1}$ requires regularization, which is implemented here using SVD (Eqs. [19]-[22]). Since usually the results very smoothly depend on the regularization parameter $q$, each resolvent can be treated with the same $q$ to reduce the number of adjusting parameters. Regularization by SVD, once again, offers the advantage of generating multiple spectra with different $q$, adding little numerical cost. Since each matrix pencil depends on one of the frequencies $s_{1}$ or $s_{2}$, the total number of SVD applications to construct the 2D spectrum on the 2D grid in the frequency domain $N_{s_{1}} \times N_{s_{2}}$ is only $N_{s_{1}}+N_{s_{2}}$. Because the most numerically expensive part of XFT is associated with SVD, the total numerical cost to construct 2D XFT scales as $\sim\left(N_{s_{1}}+N_{s_{2}}\right) \times K_{\text {win }}^{3}$, where $K_{\text {win }}$ is typically small (e.g., 100).

\subsection{Numerical Example: $2 D X F T$}

Both FDM and RRT, the predecessors of XFT, have been demonstrated previously to lead to significant resolution enhancement $(9,17)$, provided the data fit well the Lorentzian form. As shown in Fig. 1, RRT may fail when the latter is not the case (the data set is too small). In this case very strong regularization (large $q$ ) is needed to remove the artifacts and correct the lineshapes, eventually leading to a low-resolution spectrum that may become even inferior to the DFT spectral estimate. Unlike the FDM and RRT, the regularization in XFT does not generally reduce the resolution below that of the DFT; i.e., it is essentially bounded from below by the FT uncertainty principle. Usually a compromise, corresponding to some finite value of $q$, exists, leading to the Gibbs oscillation removal and resolving some peaks (not necessarily all of them) that can be fitted by Lorentzians. This is demonstrated in Fig. 4 where we present another more challenging numerical test. Each of the XFT spectra shown for different values of $q$ offer a resolution enhancement over the DFT, albeit the lineshapes are distorted for smaller values of $q$. With $q=0.001$, the regularization is optimal, and the splittings are resolved without any artifacts present. The results of two LP spectra, calculated using the NMRPipe program (21), are presented as a comparison to the XFT. As XFT does not use the data from the entire constant time period, it is compared to a standard LP algorithm. However, for constant time data, mirror-image LP (MI-LP)(22) is commonly employed and is also presented. Both LP spectra show an improvement over the FT, but some peaks that are resolved by the XFT are not improved. Recently, using ideas similar to MI-LP, FDM has been implemented to use data from the entire constant time period $2 T$ (23), which effectively doubles the data size. This led to a substantial resolution enhancement. Although this method has not yet been implemented for XFT, once it is implemented, we expect a further improvement. 

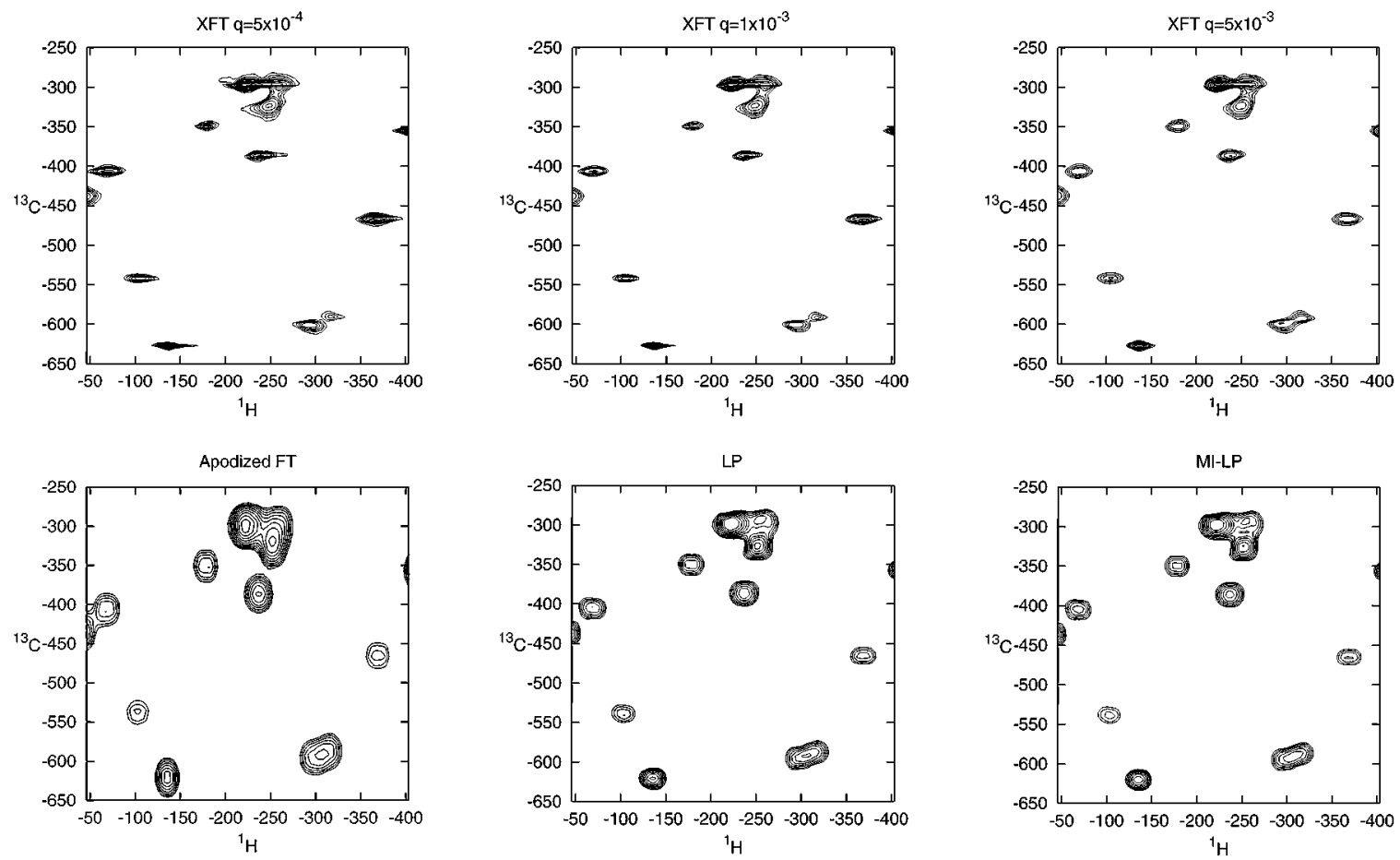

FIG . 4. Comparison of the XFT of a small region of the carbon-hydrogen CT-HSQC of Ubiquitin with the FT and LP methods. The spectra were processed for several values of $q$ to illustrate the behavior of the method. The XFT and FT spectra were processed using the entire data set $\left(N_{1}=316\right.$ and $\left.N_{2}=2048\right)$ with a spectral width of $350 \mathrm{~Hz}$ in the proton dimension and $400 \mathrm{~Hz}$ in the carbon dimension. The calculation was performed for 200 spectral points in each dimension, and for the XFT, $K_{\text {win }}=60$. The FT and LP spectra were apodized with a cosine-squared function. The LP and the MI-LP are 16th order, calculated using the NMRPipe program.

\section{SUMMARY}

XFT has been presented as a useful spectral estimation method. The fact that XFT is a function of the regularization parameter $q$ has the advantage of being able to tune the spectrum between high-resolution spectral estimation and the finite DFT. In the case where the data satisfy the Lorentzian assumption [4] (or its 2D analogue), infinitesimally small regularization is required and the XFT gives essentially the exact infinite time DFT. If the Lorentzian assumption is not met, in the case of a very short, or noisy signal, the level of resolution is controlled by adjusting the regularization. Only having a few parameters that need to be adjusted, and having a broad range over which the parameters give usable results, also makes XFT much easier to use than most other methods. XFT has numerous similarities to the RRT, albeit the nonlinear contribution (the correction term) in the former is generally relatively small. For this reason, problems that may severely affect the performance of either FDM or RRT may only cause small problems in the XFT.

The main disadvantage of the XFT is that, unlike FDM, individual resonances are not calculated and cannot be manipulated afterward. While RRT can generate other spectral representations (not necessarily the infinite time DFT spectral estimate), XFT is restricted solely to the DFT spectral estimation. Also, due to many resolvent matrix elements to be computed in XFT (see Eq. [36]), it is numerically more intensive than RRT, or at least requires more intelligent programming.

\section{APPE NDIX}

\section{Alternative Expressions to E valuate 1D XFT}

Here we rewrite Eqs. [7] and [9] for variable truncation size $N=m+m^{\prime}$

$$
\begin{aligned}
I(s) & =\sum_{n=0^{+}}^{m+m^{\prime}-1} c(n) z^{-n}+\left(\Phi_{m}\left|\frac{z^{-\left(m+m^{\prime}\right)}}{1-\hat{U} / z}\right| \Phi_{m^{\prime}}\right) \\
& =\operatorname{DFT}_{m+m^{\prime}}+\Delta_{m+m^{\prime}},
\end{aligned}
$$

which holds for any $m$ and $m^{\prime}$. We can now average $I(s)$ over these two free parameters. This leads, in principle, to an infinite number of possible expressions, depending on the weighting function. Interestingly, for the ideal data, i.e., satisfying Eq. [4], any such expression would be numerically exact, while the difference would be observed in practical applications using nonperfect signals. Here we derive just one such expression that is 


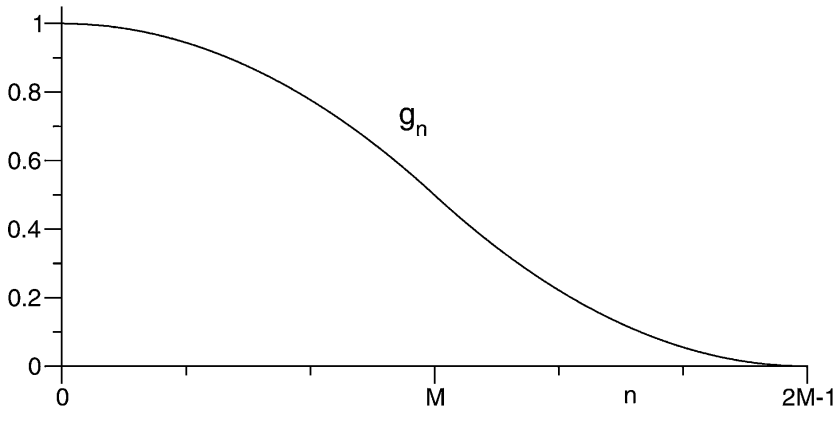

FIG. 5. The weighting function $g_{n}$, used in the averaged XFT DFT term.

compact and numerically efficient,

$$
\begin{aligned}
I(s) & =\frac{1}{(M+1)^{2}} \sum_{m=0}^{M} \sum_{m^{\prime}=0}^{M}\left\{\mathrm{DFT}_{m+m^{\prime}}+\Delta_{m+m^{\prime}}\right\} \\
& =\mathrm{DFT}_{\mathrm{av}}+\Delta_{\mathrm{av}},
\end{aligned}
$$

where the first term simply becomes an apodized DFT

$$
\mathrm{DFT}_{\mathrm{av}}=\sum_{n=0^{+}}^{2 M-1} z^{-n} c(n) g_{n}
$$

with weighting function $g_{n}$ given by

$$
\begin{aligned}
g_{n} & =1-\frac{(n+1)(n+2)}{2(M+1)^{2}}, \quad n \leq M-1 \\
& =\frac{(2 M-n)(2 M-n+1)}{2(M+1)^{2}}, \quad n \geq M,
\end{aligned}
$$

shown in Fig. 5, which has a strong apodizing effect. On its own, $g_{n}$ might not be an optimal weighting function, but in XFT the corresponding resolution loss can be compensated for. The correction term after averaging becomes

$$
\Delta_{\mathrm{av}}=\mathbf{C}_{\mathrm{av}}^{\mathrm{T}} \mathbf{R}^{-1} \mathbf{C}_{\mathrm{av}}
$$

with

$$
\mathbf{C}_{\mathrm{av}}=\frac{1}{(M+1)} \sum_{m=0}^{M} z^{-m} \mathbf{C}_{m}
$$

and $\mathbf{C}_{m}$ is defined by Eq. [10].

In the Fourier basis the correction becomes

$$
\Delta_{\mathrm{av}}=\tilde{\mathbf{C}}_{\mathrm{av}}^{\mathrm{T}} \tilde{\mathbf{R}}^{-1} \tilde{\mathbf{C}}_{\mathrm{av}}
$$

where the expression for $\tilde{\mathbf{R}}$ is unaffected by averaging and the elements of $\tilde{\mathbf{C}}_{\mathrm{av}}$ are obtained by recognizing that its form is similar to that of $\tilde{\mathbf{U}}_{0}$ in Eq. [14] upon substitution of $y_{j^{\prime}}$ by $z$ :

$$
\begin{aligned}
{\left[\tilde{\mathbf{C}}_{\mathrm{av}}\right]_{j} \equiv } & \tilde{\mathbf{U}}_{0}\left(z, y_{j}\right) \\
= & \sum_{\sigma=0,1} \frac{(-1)^{\sigma}\left(y_{j} / z\right)^{\sigma(M+1)}}{1-y_{j} / z} \sum_{n=\sigma(M+1)}^{\sigma M+M-1} y_{j}^{-n} c(n) \\
& +\sum_{\sigma=0,1} \frac{(-1)^{\sigma}\left(z / y_{j}\right)^{\sigma M}}{1-z / y_{j}} \sum_{n=\sigma M}^{\sigma(M-1)+M} z^{-n} c(n) .
\end{aligned}
$$

This result can easily be generalized to the $2 \mathrm{D}$ case, which is not done here.

\section{ACKNOWLEDGMENTS}

This work was supported by the NSF. G.S.A. and V.A.M. acknowledge grant CHE-0108823, and would like to thank Jianhan Chen and A. J. Shaka for insightful discussions. G.S.A. also thanks Anna De Angelis for providing the 2D NMR data.

\section{REFERENCES}

1. S. Marple, Jr., "Digital Spectral Analysis with Applications," Prentice-Hall, Englewood Cliffs, NJ (1987).

2. J. Tang and J. R. Norris, LPZ spectral analysis using linear prediction and the z-transform, J. Chem. Phys. 84, 5210-5211 (1986).

3. G. Zhu and A. Bax, Two-dimensional linear prediction for signals truncated in both dimensions, J. Magn. Reson. 98, 192-199 (1992).

4. H. Barkhuysen, R. de Beer, W. M. M. J. Bovee, and D. van Ormondt, Retrieval of frequencies, amplitudes, damping factors, and phases from timedomain signals using a linear least-squares procedure, J. Magn. Reson. 61, 465-481 (1985).

5. J. Tang and J. Norris, LP-ZOOM, a linear prediction method for local spectral analysis of NMR signals, J. Magn. Reson. 79, 190-196 (1988).

6. Y. Hua and T. K. Sarkar, Matrix pencil method for estimating parameters of exponentially damped/undamped sinusoids in noise, IEEE Trans. Acoust. Signal Speech Process. 38, 814-824 (1990).

7. J. C. Hoch and A. S. Stern, "NMR Data Processing," Wiley-Liss, New York (1996).

8. V. A. Mandelshtam, FDM: The filter diagonalization method for data processing in NMR experiments, Prog. Nucl. Magn. Reson. Spectrosc. 38, 159196 (2001).

9. M. R. Wall and D. Neuhauser, Extraction, through filter-diagonalization, of general quantum eigenvalues or classical normal mode frequencies from a small number of residues or a short-time segment of a signal. I. Theory and application to a quantum-dynamics model, J. Chem. Phys. 102, 8011-8022 (1995).

10. V. A. Mandelshtam and H. S. Taylor, Harmonic inversion of time signals and its applications, J. Chem. Phys. 107, 6756-6769 (1997).

11. V. A. Mandelshtam and H. S. Taylor, Multidimensional harmonic inversion by filter-diagonalization, J. Chem. Phys. 108, 9970-9977 (1998).

12. V. A. Mandelshtam, The multidimensional filter diagonalization method. I. Theory and numerical implementation, J. Magn. Reson. 144, 343-356 (2000).

13. A. A. De Angelis, H. Hu, V. A. Mandelshtam, and A. J. Shaka, The multidimensional filter diagonalization method. II. Applications to 2D, 3D and 4D NMR experiments, J. Magn. Reson. 144, 357-366 (2000). 
14. J. Chen and V. A. Mandelshtam, Multiscale filter diagonalization method for spectral analysis of noisy data with nonlocalized features, J. Chem. Phys. 112, 4429-4437 (2000).

15. J. Chen, V. A. Mandelshtam, and A. J. Shaka, Regularization of the filter diagonalization method: FDM2k, J. Magn. Reson. 146, 363-368, (2000).

16. S. D. Silverstein and M. D. Zoltowski, The mathematical basis for element and Fourier beamspace MUSIC and root-MUSIC algorithms, Digital Signal Process. 1, 161-175 (1991).

17. J. Chen, A. J. Shaka, and V. A. Mandelshtam, RRT: The Regularized resolvent transform for high resolution spectral estimation, J. Magn. Reson. 147, 129-137 (2000).

18. A. Neumaier, Solving ill-conditioned and singular linear systems: A tutorial on regularization, SIAM Rev. 40, 636, (1998).
19. A. Tikhonov, Solution of incorrectly formulated problems and the regularization method, Sov. Math. Dokl. 4, 1035-1038 (1963); A. Tikhonov and V. Arsenin, "Solutions of Ill-posed Problems," Winston and Sons, Washington (1977).

20. A. Neumaier, private communication.

21. F. Delaglio, S. Grzesiek, G. W. Vuister, G. Zhu, J. Pfeifer, and A. Bax, NMRPipe: A multidimensional spectral processing system based on UNIX pipes, J. Biomol. NMR 6, 277-293 (1995).

22. G. Zhu and A. Bax, Improved linear prediction for truncated signals of known phase, J. Magn. Reson. 90, 405-410 (1990).

23. A. A. De Angelis, J. Chen, V. A. Mandelshtam, and A. J. Shaka, A method to obtain high resolution ${ }^{13} \mathrm{C}$ CT HSQC spectra of proteins with a short constant-time period, submitted for publication. 